\title{
Influence of high-dose oral erythromycin on feeding intolerance in preterm neonates: A randomized controlled trial
}

\author{
Maryam Saboute ${ }^{1}$, Ali Mazouri ${ }^{1}$, Fatemeh NaimiDehnavi $^{2}$, Nasrin Khalesi $^{2 *}$, Zahra Farahani $^{3}$
}

Received: 2 Feb 2017

Published: 9 Feb 2018

\begin{abstract}
Background: Feeding intolerance is a common complication in preterm neonates and is responsible for prolonged hospitalization. This study aimed at assessing the effects of high-dose oral erythromycin on feeding intolerance in preterm infants.

Methods: A randomized, double blinded, placebo-controlled trial was performed during 2014 and 2015 (Tehran-Iran). Preterm neonates aged $>14$ days, who met the feeding intolerance criteria were selected for the study and their medical records were randomly assigned into 2 groups by simple randomization. Infants in group A received $10 \mathrm{mg} / \mathrm{kg}$ oral erythromycin every 6 hours for 2 days, followed by $4 \mathrm{mg} / \mathrm{kg}$ oral erythromycin every 6 hours for 5 days; and infants in group B received placebo with the same route. The number of days until reaching complete oral feeding, day of discharge from NICU, and complications related to intervention were recorded and compared between the 2 groups. Independent samples t test, Mann-Whitney, Fischer exact test, and Chi square were used to analyze the relationships between variables. P-value less than 0.05 was considered statistically significant.

Results: A total of 20 infants in group A received erythromycin and 20 infants in group B received placebo. Erythromycin could not alter the mean volume of feeding, duration of parental feeding, length of hospitalization, and frequency of feeding discontinuity $(p>0.05)$; however, mean days to reach complete feeding in group A was significantly shorter than in group B (9.80 vs. 16.80 days; $\mathrm{p}=0.001)$.

Conclusion: High-dose erythromycin as a rescue measure with no potential adverse effect is beneficial in reducing the time taken to achieve full enteral feeding. However, more extensive investigations are needed to determine the best administration dosage.
\end{abstract}

Keywords: Erythromycin, High-dose, Feeding intolerance, Preterm neonates

Copyright $@$ Iran University of Medical Sciences

Cite this article as: Saboute M, Mazouri A, NaimiDehnavi F, Khalesi N, Farahani Z. Influence of high-dose oral erythromycin on feeding intolerance in preterm neonates: A randomized controlled trial. Med J Islam Repub Iran. 2018(9 Feb);32:9. https://doi.org/10.18869/mjiri.32.9

\section{Introduction}

Functional gastrointestinal dysmotility and enteral feeding intolerance are common complications in preterm neonates. These conditions are responsible for prolonged use of parenteral hyper alimentation, increased gastric residue, abdominal distension, constipation, regurgitation or vomiting, catheter-related nosocomial infection, cholestasis, osteopenia, poor intestinal growth, necrotizing enter colitis and prolonged hospitalization (1-4).

Immaturity of gastrointestinal motility and phase III activity of the migrating motor complex (MMC) were proposed as the main causes of dysmotility and enteral feeding intolerance. On the other hand, mottling agonists by binding to mottling GI receptors would be beneficial in

Corresponding author:Dr Nasrin Khalesi, nasrinkhalessi@yahoo.com

1. Department of Pediatrics, Akbarabadi Hospital, Iran University of Medical Sciences, Tehran, Iran.

2. Aliasghar Hospital, Iran University of Medical Sciences, Tehran, Iran.

3. Maternal, Fetal and Neonatal Research Center, Tehran University of Medical Sciences, Tehran, Iran. the treatment and alleviation of this gastrointestinal problem $(1,5)$.

Prokinetic agents, such as erythromycin, induce intense motor activity in the stomach and small intestine, which mimics phase III MMC activity. Migrating motor complexes are phasic contractions originating in the proximal small intestine during fasting that propagate distally and propel the intestinal contents towards the colon (6). This GI contractile complex develops after 32 weeks of gestation $(7,8)$.

Several studies pointed to the influence of erythromycin on the treatment of gastrointestinal dysmotility and the decrease in the incidence of parenteral nutrition-associated

$\uparrow$ What is "already known" in this topic:

There are conflicting results regarding the use of prophylactic or rescue administration of different doses of oral erythromycin in preterm infants.

$\rightarrow$ What this article adds:

This study aimed at assessing the effects of $40 \mathrm{mg} / \mathrm{kg} / \mathrm{day}$ oral erythromycin on feeding intolerance in preterm infants. 
cholestasis $(9,10)$. However, a systematic review revealed more positive effects of erythromycin in improving feeding intolerance at higher doses $(40$ to 50 $\mathrm{mg} / \mathrm{kg} /$ day) or in older neonates (gestational age $>32$ weeks) (11).

Recent studies have shown different responses and conflicting results regarding the use of prophylactic or rescue administration of different doses of oral erythromycin in preterm infants $(2,8,10-12)$. This study aimed at assessing the effects of $40 \mathrm{mg} / \mathrm{kg} /$ day oral erythromycin on feeding intolerance in preterm infants and the values of some related factors.

\section{Methods}

A randomized, double blinded, placebo-controlled trial on 46 newborns with feeding intolerance was conducted in the NICU unit of Aliasghar hospital affiliated to Iran University of Medical Sciences (Tehran-Iran) during 2014 and 2015. Preterm neonates age $<32$ weeks or birth weight $<1800$ gram) aged $\geq 14$ days, who met the feeding intolerance criteria and were in a stable condition, with normal blood pressure, no bradycardia or hypoxic episodes, were selected for this study. Exclusion criteria were as follow: missing data; congenital malformation ;metabolic disorders; cyanotic heart disease; sepsis; NEC; obstructive GI disease; recent GI surgeries; or any treatment for food intolerance (Indomethacin, Fentanyl, and Pancuronium). Feeding intolerance was considered with the following criteria: enteral feeding less than $75 \mathrm{~mL} / \mathrm{kg}$ /day by Day 14 postnatal age, or gastric residual $>50 \%$ from 3 hours ago, more than 2 consecutive feeds during 24 hours, or gastric residual $>30 \%$ from 3 hours ago, more than 3 consecutive feeds during 24 hours. All infants' demographic data, including sex, age, gestational age, and type of delivery, were recorded in some checklists. Newborns' medical records were randomly assigned into 2 groups. We randomly allocated the 2 groups by Excel software using Rand Between order. Allocation into each group was based on opening sealed and opaque envelopes indicating the drug name. Clinicians and infants were blinded to the treatment allocation; infants in group A received $10 \mathrm{mg} / \mathrm{kg}$ oral erythromycin every 6 hours for 2 days, followed by 4 $\mathrm{mg} / \mathrm{kg}$ oral erythromycin every 6 hours for 5 days. Infants in group B received placebo (normal saline) with the same route. Erythromycin ethyl succinate suspension $(10 \mathrm{Mg}$ $15 \mathrm{cc}$ without any additives) and placebo without erythromycin with $1 \mathrm{cc}$ distilled water were prepared. To mask the difference in appearance (shape, color, and odor) of erythromycin and placebo, both of them were mixed in milk. Before and 5 days after the intervention, EKG was conducted and hepatic enzymes were assessed for all participants. Both nurses and attending physicians were blinded about groups and medications. Intervention was stopped after a week. The number of days until reaching complete oral feeding, day of discharge from NICU, complications related to intervention, such as diarrhea, vomiting, pyloric stenosis, and necrotizing enterocolitis (NEC) were recorded. Data of the 2 groups were compared to test the hypothesis that whether the intervention program makes any changes in the groups' feeding tolerance (tolerance of 150 $\mathrm{mL} / \mathrm{kg} /$ day) (Fig. 1). Registration ID of this study in Iranian Registry of Clinical Trials was

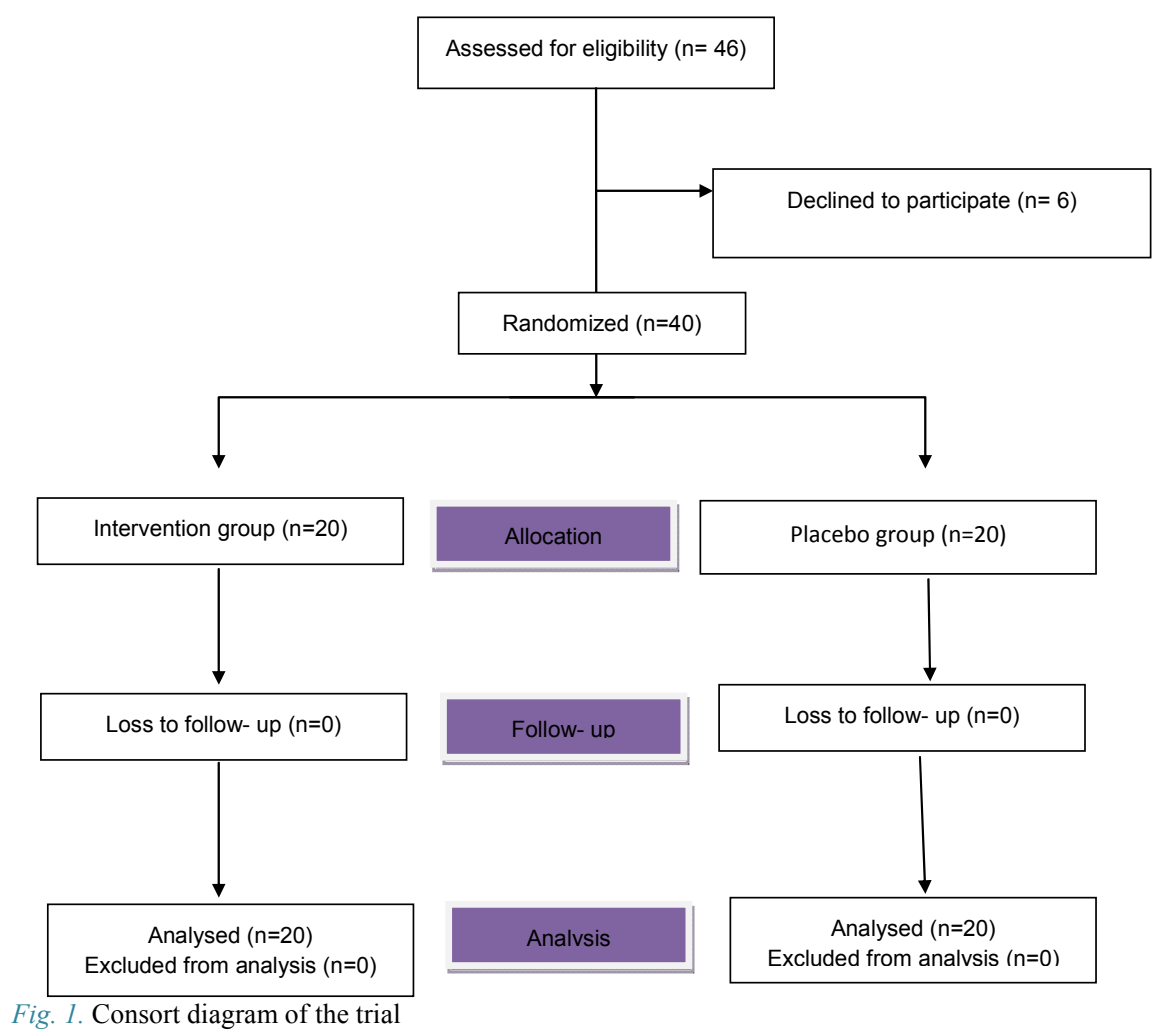




\section{IRCT2015121925591N1.}

\section{Statistical analysis}

All statistical analyses were conducted using SPSS 19. Data were presented as mean \pm standard deviation for continuous variables and $n(\%)$ for categorical variables. Independent samples t-test, Mann-Whitney, Fischer exact test, and Chi square were used to analyze the relationships between variables. P-value less than 0.05 was considered statistically significant.

Participants' parents gave informed consent before entering the study. Our data were confidential and no extra cost was constrained on our participants. Our study was approved by the institutional review board of Iran University of Medical Sciences and according to Helsinki declaration.

\section{Results}

Of 46 neonates, 6 cases were excluded because of intervention intolerance, unstable condition, or missing data. A total of 20 infants in group A received erythromycin and 20 infants in group B received placebo. The basic demographic data are demonstrated in Table 1.

In group A, 7 cases (35\%) had inadequate feeding, 4 $(20 \%)$ had gastric residue, and $9(45 \%)$ had both problems, while these numbers in group B were $3(15 \%), 7$ $(35 \%)$, and $10(50 \%)$, respectively $(\mathrm{p}=0.291)$. No significant differences were obtained between the 2 groups regarding demographic characteristics $(p>0.05)$, but patent ductus arteriosus and intraventricular hemorrhage were more frequent in group A than in group B $(p=.035)$. Erythromycin could not alter the mean volume of feeding, duration of parental feeding, length of hospitalization, and frequency of feeding discontinuity ( $p>0.05)$; however, the mean days to reach complete feeding in group A was significantly shorter than in group B (9.80 vs. 16.80 days; $\mathrm{p}=0.001)$. Regarding complications related to intervention, a neonate in each group showed NEC. Details are demonstrated in Table 2.

\section{Discussion}

In this study, we found beneficial effects of administration of high-dose oral erythromycin on neonatal feeding intolerance. Recent studies have also revealed that oral erythromycin increases endogenous motilin and activates motilin pathway through neural mechanism and smooth muscle motilin receptors. This activation results in facilitating more frequent, higher amplitude contractions in antral and proximal small bowel and declines the outlet resistance in pyloric sphincter $(2,4,13)$.

Despite immaturity in GI tract and lack of a welldeveloped migrating contractile activity in infants younger than 32 weeks of gestation, our results indicated that preterm infants achieved full oral feeding significantly earlier after intervention with oral erythromycin (9.80 vs. 16.80 days; $\mathrm{p}<0.05)$. Ng et al. in 2001 and 2007 found that the mean and median days to achieve full oral feeding in neonates with $12.5 \mathrm{mg} / \mathrm{kg}$ oral erythromycin every 6 hours were significantly shorter than neonates in placebo group (10-13). In another study, Mohammadizadeh et al. also confirmed this finding with low dose $(6 \mathrm{mg} / \mathrm{kg} /$ Day $)$ of erythromycin; the mean time taken to reach full enteral feeding in erythromycin and placebo groups was $10.11 \pm 2.51$ and $12.71 \pm 5.76$ days, respectively $(\mathrm{p}=0.01)$ (2).Yan-Yan also showed the efficacy of intermediatedose ( $5 \mathrm{mg} / \mathrm{kg} / 6$ hours) oral erythromycin on the earlier full enteral feeding among VLBW infants (36.5 days in the erythromycin group vs. 54.7days in the control group, $\mathrm{p}=0.01$ ) (14). On the other hand, $\mathrm{Ng}$ in another study found that administration of low-dose erythromycin $(5 \mathrm{mg} / \mathrm{kg}$ every 8 hours) in preterm infants with feeding intolerance could not significantly alter the mean time to achieve full enter-

Table 1. Demographic data of all participants

\begin{tabular}{|c|c|c|c|}
\hline Variable & $\begin{array}{l}\text { Erythromycin } \\
(\mathrm{N}=20)\end{array}$ & $\begin{array}{l}\text { Placebo } \\
(\mathrm{N}=20)\end{array}$ & $\mathrm{p}$ \\
\hline Male $[\mathrm{n}(\%)]$ & $11(55 \%)$ & $5(25 \%)$ & 0.105 \\
\hline Age (day) & $24.6 \pm 17.3$ & $24.4 \pm 14.4$ & 0.969 \\
\hline Weight (gr) & $1239.1 \pm 292.8$ & $1309.1 \pm 286.1$ & 0.450 \\
\hline Gestational age (weeks) & $29.65 \pm 1.56$ & $30.51 \pm 3.59$ & 0.592 \\
\hline First min apgar & $6.30 \pm 2.15$ & $8.01 \pm 0.61$ & 0.266 \\
\hline 5 min apgar & $8.30 \pm 1.34$ & $8.95 \pm 1.27$ & 0.125 \\
\hline Type of delivery; Cesarean [n (\%)] & $16(80)$ & $17(85)$ & 0.677 \\
\hline Betamethasone administration [n (\%)] & $4(20)$ & $2(10)$ & 0.376 \\
\hline $\mathrm{FIO} 2$ & $89.45 \pm 20.38$ & $92.45 \pm 14.82$ & 0.965 \\
\hline PDA/IVH [n (\%)] & $4(20)$ & 0 & 0.03 \\
\hline Umbilical catheterization $[\mathrm{n}(\%)]$ & 0 & $1(\%)$ & 0.311 \\
\hline Formula feeding $[\mathrm{n}(\%)]$ & $16(80)$ & $14(70)$ & 0.465 \\
\hline Feeding rate (cc/day) & $41.89 \pm 33.05$ & $38.72 \pm 17.47$ & 0.733 \\
\hline \multicolumn{4}{|c|}{ Table 2. Comparison of clinical variables in the erythromycin and placebo groups } \\
\hline Variables & $\begin{array}{c}\text { Erythromycin } \\
\mathrm{N}(\%)\end{array}$ & $\begin{array}{c}\text { Placebo } \\
\mathrm{N}(\%)\end{array}$ & $\mathrm{p}$ \\
\hline Reaching complete oral feeding & $5.80 \pm 9.80$ & $6.70 \pm 16.80$ & 0.001 \\
\hline Parental feeding (day) & $14.07 \pm 28.25$ & $20.46 \pm 31.01$ & 0.623 \\
\hline Length of hospitalization & $18.77 \pm 42.80$ & $18.58 \pm 42.75$ & 0.993 \\
\hline Frequency of stop feeding & $1.50 \pm .95$ & $0.83 \pm 0.81$ & 0.383 \\
\hline Complications & & & 0.999 \\
\hline NEC & $1(5)$ & $1(5)$ & \\
\hline
\end{tabular}


al feedings (24.9 days in the erythromycin group vs. 30.8 days in the placebo group, $p=0.17)(15)$. This diversity of results may be due to differences in the administration of doses, duration of intervention, or neonatal characteristics, such as weight and gestational age.

The results revealed that administration of high- dose oral erythromycin did not cause any significant potential adverse effects; a neonate with NEC was observed in each group $(p>0.05)$. Woon So et al. reported that of 10 randomized controlled trials, none found any major side effects related to oral erythromycin (3). This finding was also confirmed by $\mathrm{Ng}$; based on evidence from some RCTs in a systematic review, he found no significant association among oral erythromycin with hypertrophic pyloric stenosis, fatal cardiac arrhythmia, prolonged QTc intervals, emergence of multidrug-resistant organisms, NEC or significant alteration of the pattern of stool microflora (4).

This study found no significant difference between the 2 groups in the mean volume of feeding, duration of parental feeding, length of hospitalization, and frequency of feeding discontinuity $(\mathrm{p}>0.05)$. Aly et al. indicated no significant correlations among erythromycin with fewer episodes of gastric residuals, length of hospital stay, or duration of parenteral nutrition in preterm infants $(\mathrm{p}>0.05)$ (5). On the other hand, $\mathrm{Ng}$ et al. demonstrated a shorter duration of parenteral nutrition by 10 days in treated VLBW infants with high-dose oral erythromycin $(\mathrm{p}<0.001)$ (10).

Our study had some limitations. We did not assess the serum drug concentration achieved by oral medication, which could have provided us with some informative and beneficial data. Furthermore, conducting other studies with larger sample sizes is strongly recommended.

\section{Conclusion}

In summary, our results confirmed previous studies that indicated high-dose erythromycin for 7 days as a rescue measure with no potential adverse effect is beneficial in reducing the time taken to achieve full enteral feeding among preterm infants. However, more extensive investigations are needed to determine the best administration dosage and ensure its safety in preterm infants.

\section{Acknowledgements}

The authors would like to express their gratitude to Ali Asghar hospital NICU faculties and staff for their kindly collaboration.

\section{Conflict of Interests}

The authors declare that they have no competing interests.
2010;51(12):952

3. So KW, Ng PC. Erythromycin and gastrointestinal dysmotility in preterm infants. East J Med. 2010;15(4):146.

4. Ng PC. Use of oral erythromycin for the treatment of gastrointestinal dysmotility in preterm infants. Neonatology. 2008;95(2):97-104.

5. Aly H, Abdel-Hady H, Khashaba M, El-Badry N. Erythromycin and feeding intolerance in premature infants: a randomized trial. J Perinatol. 2007;27(1):39-43.

6. Simkiss D, Adams I, Myrdal U, Booth I. Erythromycin in neonatal postoperative intestinal dysmotility. Arch Dis Childhood-Fetal Neonatal Edit. 1994;71(2):F128-F9.

7. Jadcherla SR, Klee G, Berseth CL. Regulation of migrating motor complexes by motilin and pancreatic polypeptide in human infants. Pediatr Res. 1997;42(3):365-9.

8. Tomomasa T, Miyazaki M, Koizumi T, Kuroume T. Erythromycin increases gastric antral motility in human premature infants. Neonatology. 1993;63(6):349-52.

9. Isaacs D, Royle JA, Infections ASGfN. Intrapartum antibiotics and early onset neonatal sepsis caused by group B Streptococcus and by other organisms in Australia. Pediatr Infect Dis J. 1999;18(6):524-8.

10. Ng PC, Lee CH, Wong SP, Lam HS, Liu FY, So KW, et al. Highdose oral erythromycin decreased the incidence of parenteral nutritionassociated cholestasis in preterm infants. Gastroenterology. 2007;132(5):1726-39

11. Ng E, Shah VS. Erythromycin for the prevention and treatment of feeding intolerance in preterm infants. The Cochrane Library. 2008.

12. Oei J, Lui K. A placebo-controlled trial of low-dose erythromycin to promote feed tolerance in preterm infants. Acta Paediatrica. 2001;90(8):904-8.

13. Ng P, So K, Fung K, Lee C, Fok T, Wong E, et al. Randomised controlled study of oral erythromycin for treatment of gastrointestinal dysmotility in preterm infants. Arch Dis Childhood-Fetal and Neonatal Edit. 2001;84(3):F177-F82.

14. Ng YY, Su PH, Chen JY, Quek YW, Hu JM, Lee IC, et al. Efficacy of intermediate-dose oral erythromycin on very low birth weight infants with feeding intolerance. Pediatr Neonatol. 2012;53(1):34-40.

15. Ng SCY, Gomez JM, Rajadurai VS, Saw SM, Quak SH. Establishing enteral feeding in preterm infants with feeding intolerance: a randomized controlled study of low-dose erythromycin. J Pediatr Gastroenterol Nutri. 2003;37(5):554-8.

\section{References}

1. Asadollahpour F, Yadegari F, Soleimani F, Khalesi N. The Effects of Non-Nutritive Sucking and Pre-Feeding Oral Stimulation on Time to Achieve Independent Oral Feeding for Preterm Infants. Iran J Pediatr. 2015;25(3):809.

2. Mohammadizadeh M, Ghazinour M, Iranpour R. Efficacy of prophylactic oral erythromycin to improve enteral feeding tolerance in preterm infants: A randomised controlled study. Singapore Med J. 